\title{
ENTROPY SOLUTIONS FOR DIFFUSION-CONVECTION EQUATIONS WITH PARTIAL DIFFUSIVITY
}

\author{
M. ESCOBEDO, J. L. VAZQUEZ, AND ENRIKE ZUAZUA
}

\begin{abstract}
We consider the Cauchy problem for the following scalar conservation law with partial viscosity

$$
u_{t}=\Delta_{x} u+\partial_{y}(f(u)), \quad(x, y) \in \mathbf{R}^{N}, t>0 .
$$

The existence of solutions is proved by the vanishing viscosity method. By introducing a suitable entropy condition we prove uniqueness of solutions. This entropy condition is inspired by the entropy criterion introduced by Kruzhkov for hyperbolic conservation laws but it takes into account the effect of diffusion.
\end{abstract}

\section{INTRODUCTION}

We present a theory of existence and uniqueness for suitable entropy solutions of a nonlinear equation which describes the combined effects of diffusion and convection of matter. It has the form

$$
u_{t}=\Delta_{x} u+\partial_{y} f(u)
$$

posed in the space $\mathbf{R}^{N}, N \geq 2$, denoted by the variable $z=(x, y), x \in \mathbf{R}^{N-1}$, $y \in \mathbf{R}$. The main characteristic of this equation is that it has mixed parabolichyperiolic type, due to the directional separation of the diffusion and convection effects: while matter is convected along the $y$ axis, it is simultaneously diffused along all orthogonal directions.

Our interest in the model is not merely academic. We have encountered it while studying in [EVZ] the asymptotic behavior of a 'standard' nonlinear diffusion-convection model of the form

$$
u_{t}=\Delta_{z} u+\partial_{y} f(u) \text {, }
$$

in the case $f(u)=u|u|^{q-1}$ for $1<q<(N+1) / N$. Notice that $\Delta_{z} u=$ $\Delta_{x} u+\partial_{y}^{2} u$. In fact, certain self-similar solutions of equation (0.1) will give the large-time behaviour of the nonnegative solutions of $(0.2)$ having finite mass. The unique characterization of solutions of $(0.1)$ is essential in establishing the asymptotic behaviour of $(0.2)$. The comparison of both equations suggests the name 'reduced equation' for (0.1).

The existence of solutions of equation (0.1) can be obtained by the classical method of adding a vanishing artificial viscosity, in other terms a diffusion, in

Received by the editors December 21, 1992.

1991 Mathematics Subject Classification. Primary 35L65, 35K65, 35A05.

Key words and phrases. Convection-diffusion equations, scalar conservation laws, partial diffusivity, entropy criterion, uniqueness, vanishing viscosity. 
the missing direction (along the $y$-axis). We consider the existence theory for the Cauchy problem with bounded and integrable initial data.

The main interest of our treatment lies in the uniqueness part. It is well known that solutions of conservation laws are not characterized in a unique way unless we impose extra conditions, so-called entropy conditions. Such problems arise for equation $(0.1)$. In dealing with uniqueness we will be inspired in the entropy conditions in the form given by Kruzhkov, [Kr], which is now classical. However, in view of the presence of the diffusion term $\Delta_{x} u$ in equation $(0.1)$ Kruzhkov's entropy criterion has to be modified. This is done by introducing as entropy test functions all functions of the form $|u-\psi(x)|$ and $\psi$ smooth, while Kruzhkov's definition asks for $\psi$ to be constant. This change also implies the modification of the criterion formula which now reads for a candidate solution $u(x, y, t)$ :

$$
\begin{aligned}
\frac{\partial}{\partial t} \mid u & -\psi(x)\left|-\Delta_{x}\right| u-\psi(x) \mid \\
& \leq \frac{\partial}{\partial y}[(f(u)-f(\psi(x))) \operatorname{sign}(u-\psi)]+\operatorname{sign}(u-\psi(x)) \Delta_{x} \psi(x),
\end{aligned}
$$

which is to be understood in the sense of distributions. The function sign is defined as $\operatorname{sign}(s)=1$ for $s>0,-1$ for $s<0$ and 0 for $s=0$. In this setting uniqueness is established for entropy solutions of the Cauchy problem with initial data in $L^{1} \cap L^{\infty}$.

Most of the tools we use are well known in the theory of viscous or hyperbolic scalar conservation laws. However, equation $(0.1)$ presents important new difficulties due to its mixed hyperbolic-parabolic character depending on directions.

Before proceeding with the proofs, let us make some comments. Equation (0.1) admits as solutions functions of the form $u(y, t)$ as long as they are solutions of the nonviscous conservation law $u_{t}=\partial_{y} f(u)$. This points out the need for some kind of entropy condition in equation $(0.1)$, notwithstanding the fact that a viscosity term is present. On the other hand, solutions of the form $u=u(x, t)$ coincide with the solutions of the heat equation $u_{t}=\Delta_{x} u$, where no such additional condition is needed. In fact, the restriction of condition (EC) to such equations holds as a consequence of the regularity of the solutions.

The condition we suggest modifies Kruzhkov's condition to take into account those facts.

\section{Statement of the Main Results}

We will study the existence, uniqueness and properties of entropy solutions for the Cauchy problem associated to the diffusion-convection equation of the reduced type

$$
\begin{aligned}
& u_{t}=\Delta_{x} u+\partial_{y} f(u), \\
& u(x, 0)=u_{0}(x, y),
\end{aligned}
$$

with $f$ a locally Lipschitz continuous real function such that $f(0)=0$ and initial data $u_{0} \in L^{1}\left(\mathbf{R}^{N}\right) \cap L^{\infty}\left(\mathbf{R}^{N}\right)$.

Definitions. (i) By a solution of (1.1) we will understand a function

$$
u \in C\left((0, \infty): L^{1}\left(\mathbf{R}^{N}\right)\right) \cap L^{\infty}(Q), \quad Q=\mathbf{R}^{N} \times(0, \infty),
$$

which satisfies $(1.1)$ in the sense of distributions. 
(ii) A solution of problem (1.1)-(1.2) is a solution of $(1.1)$ such that $u(\cdot, t) \rightarrow$ $u_{0}$ in $L^{1}\left(\mathbf{R}^{N}\right)$ as $t \rightarrow 0$. Thus, $u=u(x, y, t)$ is continuous at $t=0$ as a function: $[0, \infty) \mapsto L^{1}\left(\mathbf{R}^{N}\right)$.

(iii) A solution $u(x, y, t)$ is called entroy solution if the entropy criterion (EC) is satisfied for all smooth functions $\psi=\psi(x)$. As said above, it extends Kruzhkov's definition by allowing $\psi$ to be nonconstant. This forces us to introduce the extra term

$$
\operatorname{sign}(u-\psi(x)) \Delta_{x} \psi(x)
$$

on the right-hand side, and the term $-\Delta_{x}|u-\psi(x)|$ on the left-hand side. Our main results are

Theorem 1. For every $u_{0} \in L^{1}\left(\mathbf{R}^{N}\right) \cap L^{\infty}\left(\mathbf{R}^{N}\right)$ there exists an entropy solution of problems (1.1)-(1.2). This solution can be constructed by the vanishing-viscosity method.

Theorem 2. The entropy solution of problems (1.1)-(1.2) is unique. Moreover, comparison holds:

$$
\text { If } u_{0} \leq v_{0} \text { a.e. in } \mathbf{R}^{N} \text {, then } u \leq v \text { in } Q=\mathbf{R}^{N} \times(0, \infty) \text {. }
$$

Finally, the following $L^{1}$-contraction property is true: if $u$ and $v$ are two entropy solutions with initial data $u_{0}, v_{0}$ resp., then for every $t>0$ :

$$
\|u(\cdot, t)-v(\cdot, t)\|_{1} \leq\left\|u_{0}-v_{0}\right\|_{1} .
$$

Moreover,

$$
\begin{aligned}
\int_{\mathbf{R}^{N-1}} & \int_{|y| \leq R}|u(x, y, t)-v(x, y, t)| d y d x \\
& \leq \int_{\mathbf{R}^{N-1}} \int_{|y| \leq R+\alpha t}\left|u_{0}(x, y)-v_{0}(x, y)\right| d y d x
\end{aligned}
$$

where

$$
\alpha=\max \left\{\left\|f^{\prime}(u)\right\|_{L^{\infty}\left(\mathbf{R}^{N} \times(0, T)\right)},\left\|f^{\prime}(v)\right\|_{L^{\infty}\left(\mathbf{R}_{x}^{N}(0, T)\right)}\right\} .
$$

The main properties of the solution are summarized as follows:

Theorem 3. Let $u$ be an entropy solution of (1.1) with initial data $u_{0} \in L^{1}\left(\mathbf{R}^{N}\right)$. Then, for every $t>0$ we have conservation of mass:

$$
\int u(x, y, t) d x d y=\int u_{0}(x, y) d x d y
$$

We also have

$$
\|u(\cdot, t)\|_{p} \leq\left\|u_{0}\right\|_{p}
$$

for every $p \in[1, \infty]$. If in addition $u_{0}$ is smooth we have further properties. Thus, if $u_{0}$ has bounded variation

$$
T V(u(\cdot, t)) \leq T V\left(u_{0}\right)
$$

and if moreover $u_{0}$ has a spatial gradient of bounded variation in the $x$ directions

$$
\left\|u\left(\cdot, t_{2}\right)-u\left(\cdot, t_{1}\right)\right\|_{1} \leq C\left[T V\left(u_{0}\right)+T V\left(\nabla_{x} u_{0}\right)\right]\left|t_{2}-t_{1}\right|,
$$

with $C=C\left(\left\|u_{0}\right\|_{\infty}\right)$. 


\section{EXISTENCE OF SOLUTIONS FOR SMOOTH DATA}

We establish here the existence part of Theorem 1 in the case where the initial data are smooth, precisely under the condition

$$
\begin{gathered}
u_{0} \in L^{\infty}\left(\mathbf{R}^{N}\right) \cap B V\left(\mathbf{R}^{N}\right) \cap L^{1}\left(\mathbf{R}^{N} ; 1+|x|+|y|\right), \quad \text { and } \\
\nabla_{x} u_{0} \in\left(B V\left(\mathbf{R}^{N}\right)\right)^{N-1} .
\end{gathered}
$$

We derive at the same time the main properties of the solution as listed above.

The construction of the solution uses the classical vanishing-viscosity method. Define $u_{0, \varepsilon}=u_{0} * \zeta_{\varepsilon}$, where $*$ denotes convolution in $\mathbf{R}^{N}, \zeta_{\varepsilon}=\zeta_{\varepsilon}(x, y)=$ $\varepsilon^{-N} \zeta(x / \varepsilon, y / \varepsilon), \zeta$ being a smooth cut-off function in $\mathscr{D}\left(\mathbf{R}^{N}\right)$ with the following properties:

(i) $\zeta$ is nonnegative and its support is contained in the unit ball of $\mathbf{R}^{N}$.

(ii) The integral of $\zeta$ over $\mathbf{R}^{N}$ is 1 .

(iii) $\zeta(-x,-y)=\zeta(x, y)$.

We then consider the regularized parabolic problem

$$
\left\{\begin{array}{l}
u_{t}=\Delta_{x} u+\varepsilon \partial_{y}^{2} u+\partial_{y} f(u) \\
u(x, y, 0)=u_{0, \varepsilon}(x, y)
\end{array}\right.
$$

It is well known (cf. for instance [EZ]) that for all $\varepsilon \geq 0$ this problem has a unique solution

$$
u_{\varepsilon} \in C\left([0, \infty): L^{1}\left(\mathbf{R}^{N}\right)\right) \cap L^{\infty}(Q) .
$$

In addition $u_{\varepsilon} \in C\left(\left[0, \infty: W^{2, p}\left(\mathbf{R}^{N}\right)\right) \cap C^{1}\left([0, \infty) ; L^{p}\left(\mathbf{R}^{N}\right)\right)\right.$ for all $1<p<$ $\infty$. On the other hand, $u_{\varepsilon}$ satisfies the properties (1.3) to (1.6).

Proceeding as in the proof of Lemma 3.2, p. 68, of Godlewski and Raviart [GR], we deduce that for every $t$ and $\varepsilon>0$ :

$$
\begin{gathered}
\int u_{\varepsilon}(x, y, t) d x d y=\int u_{0, \varepsilon}(x, y) d x d y \\
\left\|\nabla u_{\varepsilon}(t)\right\|_{1} \leq\left\|\nabla u_{0, \varepsilon}\right\|_{1} \leq T V\left(u_{0}\right) \\
\left\|\partial_{t} u_{\varepsilon}(t)\right\|_{1} \leq C\left[T V\left(u_{0}\right)+T V\left(\nabla_{x} u_{0}\right)\right]
\end{gathered}
$$

On the other hand, multiplying (2.2) by $u|u|^{p-2}$ and integrating gives

$$
\left\|u_{\varepsilon}(t)\right\|_{p} \leq\left\|u_{0}\right\|_{p} \quad \text { for all } 1 \leq p \leq \infty .
$$

These estimates allow us to pass to the limit and to get a solution

$$
u \in C\left([0, \infty) ; L^{1}\left(\mathbf{R}^{N}\right)\right) \cap L^{\infty}(Q), \quad Q=\mathbf{R}^{N} \times(0, \infty),
$$

satisfying (1.6)-(1.8).

In order to prove equality (1.5), i.e. the conservation of mass for $u$, we need to prove a uniform estimate for the tails of $u_{\varepsilon}$. Taking into account that $u_{0} \in L^{1}\left(\mathbf{R}^{N} ; 1+|x|+|y|\right)$ we can prove that $u_{0 \varepsilon}$ is uniformly bounded in $L^{1}\left(\mathbf{R}^{N} ; 1+|x|+|y|\right)$. Then, by doing $L^{1}$-estimates on the equations that the $x_{i} u_{\varepsilon}$ and $y u_{\varepsilon}$ satisfy, we get that

$$
\int\left|u_{\varepsilon}(x, y, t)\right|(|x|+|y|) d x d y \leq C(t)
$$


for every $t$ and $\varepsilon>0$. This allows us to estimate the tails of $u_{\varepsilon}$ and to obtain the conservation of mass for $u$.

Finally, since $u_{\varepsilon}$ satisfies the entropy condition (EC) for all $\varepsilon>0$, passing to the limit as $\varepsilon \rightarrow 0$ we conclude that $u$ also satisfies the entropy condition.

\section{UNIQUENESS OF ENTROPY SOLUTIONS WITH SMOOTH DATA}

We establish the following result

Theorem 3.1. Let us assume that, as in the above section,

$$
u_{0} \in L^{1}\left(\mathbf{R}^{N} ; 1+|x|+|y|\right) \cap L^{\infty}\left(\mathbf{R}^{N}\right) \cap B V\left(\mathbf{R}^{N}\right), \quad \nabla_{x} u_{0} \in\left(B V\left(\mathbf{R}^{N}\right)\right)^{N-1} .
$$

Then there is a unique bounded entropy solution of problem (1.1)-(1.2). Moreover, (1.3) and (1.4) hold.

Proof. Let us denote by $u$ the solution obtained in the section above by the vanishing-viscosity method, and let $v=v(x, y, t)$ be another entropy solution with the same initial data. We will prove that $u=v$.

Remark. For notational simplicity the expression

$$
\partial_{y}[(f(u)-f(\psi)) \operatorname{sign}(u-\psi)],
$$

which appears frequently in the calculations is replaced by $\partial_{y}|f(u)-f(\psi)|$. This is only true if $f$ is nondecreasing, as our original motivation. But, except for this change the proof applies to every locally Lipschitz function real $f$.

(I) We shall take advantage of the fact that $u$ has been constructed by the vanishing-viscosity method. Indeed, since $u_{\varepsilon}$ is a solution of the regularized parabolic problem we have

$$
\left|u_{\varepsilon}-\psi\right|_{t}-\left(\Delta_{x} u_{\varepsilon}\right) \operatorname{sign}\left(u_{\varepsilon}-\psi\right)-\varepsilon \partial_{y}^{2}\left|u_{\varepsilon}-\psi\right| \leq \partial_{y}\left|f\left(u_{\varepsilon}\right)-f(\psi)\right|
$$

in $\mathscr{D}^{\prime}(Q), Q=\mathbf{R}_{x}^{N-1} \times \mathbf{R}_{y} \times \mathbf{R}_{t}^{+}$, for all $\varepsilon>0$ and all $\psi=\psi(x) \in L^{\infty}\left(\mathbf{R}^{N}\right)$. For fixed $z \in \mathbf{R}$ and $\tau \in \mathbf{R}^{+}$we now take $\psi(x)=v(x, z, \tau)$ in (3.2) to get

$$
\begin{gathered}
\left|u_{\varepsilon}(x, y, t)-v(x, z, \tau)\right|_{t}-\left(\Delta_{x} u_{\varepsilon}(x, y, t)\right) \operatorname{sign}\left(u_{\varepsilon}(x, y, t)-v(x, z, \tau)\right) \\
-\varepsilon \partial_{y}^{2}\left|u_{\varepsilon}(x, y, t)-v(x, z, \tau)\right| \leq \partial_{y}\left|f\left(u_{\varepsilon}(x, y, t)\right)-f(v(x, z, \tau))\right|
\end{gathered}
$$

in $\mathscr{D}^{\prime}(Q)$, with respect to the same variables. On the other hand, since $u_{\varepsilon}$ is smooth, we may take $\psi(x)=u_{\varepsilon}(x, y, t)$ in the entropy condition satisfied by $v=v(x, z, \tau)$. We thus get

$$
\begin{aligned}
& \left|v(x, z, \tau)-u_{\varepsilon}(x, y, t)\right|_{\tau}-\Delta_{x}\left|v(x, z, \tau)-u_{\varepsilon}(x, y, t)\right| \\
& \leq \partial_{z}\left|f(v(x, z, \tau))-f\left(u_{\varepsilon}(x, y, t)\right)\right| \\
& \quad+\Delta_{x} u_{\varepsilon}(x, y, t) \operatorname{sign}\left(v(x, z, \tau)-u_{\varepsilon}(x, y, t)\right)
\end{aligned}
$$

in $\mathscr{D}^{\prime}(Q)$, where now $Q=\mathbf{R}_{x}^{N-1} \times \mathbf{R}_{y} \times \mathbf{R}_{\tau}^{+}$. These two inequalities are the cornerstone of the proof.

(II) We now establish the main distribution inequality for differences of solutions. 
Jemma 3.2. Let $u=u(x, y, t)$ be the entropy solution of (1.1) constructed by the vanishing-viscosity method as in $\S 1$ taking initial data $u_{0}=u_{0}(x, y)$ with the assumptions (3.1). Let $v=v(x, y, t)$ be any other uniformly bounded entropy solution of (1.1). Then

$$
\partial_{t}|u-v|-\Delta_{x}|u-v|-\partial_{y}|f(u)-f(v)| \leq 0 \quad \text { in } \mathscr{D}^{\prime}(Q) .
$$

Proof. We follow familiar ground, cf. the textbook [GR]. Firstly, we take a cut-off function $r=r(y, t) \in \mathscr{D}\left(\mathbf{R} \times \mathbf{R}^{+}\right)$and set

$$
r_{\delta}(y, t)=\delta^{-2} r(y / \delta, t / \delta) .
$$

We also take a nonnegative test function, $\Phi(x, \xi, s) \in \mathscr{D}\left(\mathbf{R}^{N} \times(0, \infty)\right)$, and define two functions:

$$
\phi_{(z, \tau)}(x, y, t)=\eta_{(y, t)}(x, z, \tau)=\Phi\left(x, \frac{y+z}{2}, \frac{t+\tau}{2}\right) r_{\delta}\left(\frac{y-z}{2}, \frac{t-\tau}{2}\right) .
$$

(In the sequel the subindices $(z, \tau)$ in $\phi$ and $(y, t)$ in $\eta$ will be frequently omitted.) Using $\phi$ as test function in (3.3) and $\eta$ in (3.4) and integrating the inequalities we get with respect to $(y, t)$ and $(z, \tau)$ respectively, we obtain, with $u_{\varepsilon}=u_{\varepsilon}(x, y, t)$ and $v=v(x, z, \tau)$ :

$$
\begin{array}{r}
0 \leq \int_{0}^{\infty} \int_{0}^{\infty} \int_{\mathbf{R}} \int_{\mathbf{R}} \int_{\mathbf{R}^{N-1}}\left\{\left|u_{\varepsilon}-v\right|\left(\frac{\partial \varphi}{\partial t}+\frac{\partial \eta}{\partial \tau}\right)\right. \\
-\left|f\left(u_{\varepsilon}\right)-f(v)\right|\left(\frac{\partial \phi}{\partial y}+\frac{\partial \eta}{\partial z}\right) \\
\left.+\varepsilon\left|u_{\varepsilon}-v\right| \partial_{y}^{2} \phi+\left|u_{\varepsilon}-v\right| \Delta_{x} \eta\right\} d x d y d z d t d \tau .
\end{array}
$$

Passing to the limit as $\varepsilon \rightarrow 0$ we obtain that

$$
\begin{aligned}
0 \leq \int_{0}^{\infty} \int_{0}^{\infty} \int_{\mathbf{R}} \int_{\mathbf{R}} \int_{\mathbf{R}^{N-1}}\left\{| u - v | \left(\frac{\partial \phi}{\partial z}+\right.\right. & \left.\frac{\partial \eta}{\partial \tau}\right) \\
-|f(u)-f(v)| & \left(\frac{\partial \phi}{\partial y}+\frac{\partial \eta}{\partial z}\right) \\
& \left.+|u-v| \Delta_{x} \eta\right\} d x d y d z d t d \tau
\end{aligned}
$$

where $u=u(x, y, t)$. We not take into account that

$$
\frac{\partial \phi}{\partial t}+\frac{\partial \eta}{\partial \tau}=\frac{\partial \Phi}{\partial s}\left(x, \frac{y+2}{2}, \frac{t+\tau}{2}\right) r_{\delta}\left(\frac{y-z}{2}, \frac{t-\tau}{2}\right)
$$

and

$$
\frac{\partial \phi}{\partial y}+\frac{\partial \eta}{\partial z}=\frac{\partial \Phi}{\partial \xi}\left(x, \frac{y+z}{2}, \frac{t+\tau}{2}\right) r_{\delta}\left(\frac{y-z}{2}, \frac{t-\tau}{2}\right) .
$$

Let us introduce the change of variables

$$
Y=\frac{y+z}{2}, \quad Z=\frac{y-z}{2}, \quad T=\frac{t+\tau}{2}, \quad S=\frac{t-\tau}{2},
$$


that maps $\mathbf{R}^{2} \times\left(\mathbf{R}^{+}\right)^{2}$ into $\Omega=\left\{(Y, Z, T, S) \in \mathbf{R}^{2} \times \mathbf{R}^{2}: T+S \geq 0, T-S \geq\right.$ $0\}$. Setting

$$
\begin{aligned}
G= & G(x, Y, Z, T, S) \\
= & |u(x, Y+Z, T+S)-v(x, Y-Z, T-S)| \frac{\partial \Phi}{\partial s}(x, Y, T) \\
& -|f(u(x, Y+Z, T+S))-f(v(x, Y-Z, T-S))| \frac{\partial \Phi}{\partial \xi}(x, Y, T) \\
& +|u(x, Y+Z, T+S)-v(x, Y-Z, T-S)| \Delta_{x} \Phi(x, Y, T)
\end{aligned}
$$

we may rewrite (3.6) as follows:

$$
J_{\delta} \equiv \int_{\mathbf{R}^{N-1}} \int_{\Omega} G(x, Y, Z, T, S) r_{\delta}(Z, S) d x d Y d T d Z d S \geq 0 .
$$

We now claim that

$$
J_{\delta} \rightarrow \int_{0}^{\infty} \int_{\mathbf{R}^{N}} G(x, Y, 0, T, 0) d x d Y d T=J_{0} \quad \text { as } \delta \rightarrow 0 .
$$

But then,

$$
J_{0}=\int_{0}^{\infty} \int_{\mathbf{R}^{N}}\left\{|u-v| \frac{\partial \Phi}{\partial s}-|f(u)-f(v)| \frac{\partial \Phi}{\partial \xi}+|u-v| \Delta_{x} \Phi\right\} d x d Y d T \geq 0
$$

i.e. (3.5). In order to prove this claim we introduce the function

$$
\chi(T, \tau)= \begin{cases}1 & \text { if } T+S \geq 0, T-S \geq 0, \\ 0 & \text { otherwise }\end{cases}
$$

With this notation we have

$$
J_{\delta}=\int_{\mathbf{R}^{n+3}} G(x, Y, Z, T, S) r_{\delta}(Z, S) \chi(T, S) d x d Y d Z d T d S .
$$

On the other hand, since $\int_{\mathbf{R}^{2}} r_{\delta}(z, S) d Z d S=1$, we also have

$$
J_{0}=\int_{\mathbf{R}^{N+3}} G(x, Y, 0, T, 0) \chi(T, 0) r_{\delta}(Z, S) d x d Y d Z d T d S .
$$

Let us denote by $K$ the support of $\Phi$ and by $C_{\delta}$ the support of $r_{\delta}$. We may assume that

$$
C_{\delta} \subset\{(Z, S):|Z| \leq \delta,|\tau| \leq \delta\} .
$$

We have

$$
\begin{gathered}
\left|J_{\delta}-J_{0}\right| \leq \int_{K} d x d Y d T \int_{C_{\delta}} \mid G(x, Y, Z, T, S) \chi(T, S) \\
-G(x, Y, 0, T, 0) \chi(T, 0) \mid r_{\delta}(Z, S) d Z d S,
\end{gathered}
$$

and therefore, $\left|J_{\delta}-J_{0}\right| \leq A_{\delta}+B_{\delta}$, with

$A_{\delta}=\int_{K} d x d Y d T \int_{C_{\delta}}|G(x, Y, Z, T, S)-G(x, Y, 0, T, S)| r_{\delta}(Z, S) d Z d S$,

and

$$
B_{\delta}=\int_{K}|G(x, Y, 0, T, 0)|\left[\int_{C_{\delta}}|\chi(T, S)-\chi(T, 0)| r_{\delta}(Z, S) d Z d S\right] d Y d T d x
$$


Proceeding as in p. 75 of [GR] we deduce that

$$
\begin{aligned}
& |G(x, Y, Z, T, S)-G(x, Y, 0, T, 0)| \\
& \quad \leq C_{1}\{|u(x, Y+Z, T+S)-u(x, Y, T)| \\
& \quad+|v(x, Y-Z, T-S)-v(x, Y, T)|\}
\end{aligned}
$$

with $C_{1}=C\left(\|u\|_{L^{\infty}(Q)},\|v\|_{L^{\infty}(Q)}\right)$. Since $\left|r_{\delta}\right| \leq C_{2} \delta^{-2}$ we find

$$
\begin{aligned}
A_{\delta} \leq C_{3} \delta^{-2} \int_{K} d x d Y d T \int_{C_{\delta}} & \{|u(x, Y+Z, T+S)-u(x, Y, T)| \\
& +|v(x, Y-Z, T-S)-v(x, Y, T)|\} d Z d S .
\end{aligned}
$$

From Lebesgue's differentiation theorem we know that, for a.e. $x$,

$$
\lim _{\delta \rightarrow 0} \frac{1}{\operatorname{meas}\left(C_{\delta}\right)} \int_{C_{\delta}}|u(x, Y+Z, T+S)-u(x, Y, T)| d Z d S=0
$$

for almost all $(Y, T) \in \mathbf{R} \times \mathbf{R}_{+}$. Of course, we have an analogous result for $v$. Then, applying Lebesgue's dominated convergence theorem we deduce that:

$$
\begin{aligned}
\lim _{\delta \rightarrow 0} \delta^{-2} \int_{k} d x d Y d T \int_{C_{\delta}} & {[|u(x, Y+Z, T+S)-u(x, Y, T)|} \\
& +|v(x, Y-Z, T-S)-v(x, Y, T)|] d z d S=0,
\end{aligned}
$$

and this implies that $A_{\delta} \rightarrow 0$ as $\delta \rightarrow 0$. On the other hand,

$$
\int_{C_{\delta}}|\chi(T, S)-\chi(T, 0)| r_{\delta}(Z, S) d Z d S \leq C_{4} \delta^{-1} \int_{-\delta}^{\delta}|\chi(T, S)-\chi(T, 0)| d S,
$$

and therefore,

$$
B_{\delta} \leq C_{5} \delta^{-1} \int_{\mathbf{R}} d T \int_{-\delta}^{\delta}|\chi(T, S)-\chi(T, 0)| d S .
$$

It is then easy to check that $B_{\delta} \leq C_{5} \delta$. This concludes the proof of claim (3.8) and the lemma.

Remark 3.3. Note that in the proof of (3.5) we have not used the initial data taken by $v$ at time $t=0$. We have only used that $v \in L^{\infty}(Q)$. Of course, concerning uniqueness only the case where $v$ solves (1.1) with the same initial data as $u$ is of interest.

(III) Proceeding with the uniqueness proof, let us now formally integrate inequality (3.5) over the set $\mathbf{R}_{x}^{N-1} \times K_{R, T}$ with

$$
K_{R, T}=\left\{(y, t) \in \mathbf{R} \times \mathbf{R}^{+}:|y| \leq R+\alpha(T-t), t \in[0, T]\right\}
$$

with $\alpha=\left(\max \left\|f^{\prime}(u)\right\|_{L^{\infty}(Q)},\left\|f^{\prime}(v)\right\|_{L^{\infty}(Q)}\right)$ and $R, T>0$. We obtain that

$$
\begin{aligned}
& \int_{\mathbf{R}^{N-1}} \int_{|y| \leq R}|u(x, y, T)-v(x, y, T)| d y d x \\
& \quad-\int_{\mathbf{R}^{N-1}} \int_{|y| \leq R+\alpha T}\left|u_{0}(x, y)-v_{0}(x, y)\right| d x d y \\
& \quad+\int_{\mathbf{R}^{N-1}} \int_{\Sigma_{R, T}}\left\{|u-\varphi| n_{t}+|f(u)-f(v)| n_{y}\right\} d \sigma d x \leq 0,
\end{aligned}
$$


where $\Sigma_{R, T}=\left\{(y, t) \in \mathbf{R} \times \mathbf{R}^{+}:|y|=R+\alpha(T-t), t \in[0, T]\right\}$ is the lateral surface to $K_{R, T}$ and $n=\left(n_{y}, n_{t}\right)$ is the outward normal to $\Sigma_{R, T}$.

In (3.9) we have denoted by $u_{0}$ and $v_{0}$ the initial data of $u$ and $v$ respectively. Since

$$
|f(u)-f(v)| \geq-\alpha|u-v|
$$

we have

$$
|u-v| n_{t}+f(u)-f(v)\left|n_{y} \geq\right| u-v\left|n_{t}-\alpha\right| u-v \mid n_{y}=0
$$

on $\Sigma_{R, T}$, so that, at least formally, we get the basic dependence formula

$$
\begin{aligned}
& \int_{\mathbf{R}^{N-1}} \int_{|y| \leq R}|u(x, y, T)-v(x, y, T)| d x d y \\
& \quad \leq \int_{\mathbf{R}^{N-1}} \int_{|y| \leq R+\alpha T}\left|u_{0}(x, y)-v_{0}(x, y)\right| d y d x .
\end{aligned}
$$

Obviously, (3.10) implies in particular the uniqueness of solutions.

In the next lemma we make a precise statement about (3.10) that we will prove below:

Lemma 3.4. Let $u_{0}=u_{0}(x, y)$ be as above and $u$ the solution of (1.1) obtained by the vanishing-viscosity method. Let $v$ be any other uniformly bounded entropy solution with initial data $v_{0} \in L^{1}\left(\mathbf{R}^{N}\right) \cap L^{\infty}\left(\mathbf{R}^{N}\right)$. Then, for any $R, T>0$ we have (3.10) with

$$
\alpha=\max \left\{\left\|f^{\prime}(u)\right\|_{L^{\infty}(Q)},\left\|f^{\prime}(v)\right\|_{L^{\infty}(Q)}\right\} .
$$

Remarks 3.5. Of course, this lemma implies the uniqueness of the entropy solution of (1.1) for smooth initial data satisfying (3.1).

In addition to the uniqueness result this lemma yields the finite speed of propagation that (1.1) possesses in the direction $y$.

Proof of Lemma 3.4. We begin by approximating the characteristic function of the set $K_{R, T}$. We introduce the following approximation of Heaviside's function:

$$
Y_{\varepsilon}(t)=\int_{\infty}^{t} \zeta_{\varepsilon}(s) d s
$$

with $\zeta_{\varepsilon} \in \mathscr{D}(\mathbf{R})$ a cut-off function with support on $[-\varepsilon, \varepsilon]$. Then, for $\delta, \varepsilon$, $\theta>0$ with $\delta<T$ we set

$$
\varphi(y, t)=\left(Y_{\varepsilon}(t-\delta)-Y_{\varepsilon}(t-T)\right)\left(1-Y_{\theta}(|y|-R-M(T-t))\right) .
$$

Clearly $\varphi \in \mathscr{D}\left(\mathbf{R} \times \mathbf{R}^{+}\right)$is nonnegative and satisfies

$$
\varphi(y, t)= \begin{cases}1 & \text { if }|y| \leq R+\alpha(T-t)-\theta \text { and } \varepsilon+\delta \leq t \leq T-\varepsilon \\ 0 & \text { if }|y| \geq R+\alpha(T-t)+\theta \text { or } t \leq \delta+\varepsilon\end{cases}
$$

Let us now suppose that $\varepsilon<\delta$. Given any nonnegative $\psi=\psi(x) \in \mathscr{D}\left(\mathbf{R}^{N-1}\right)$ 
we use $\psi(x) \varphi(y, t)$ as a test function in (3.5) to get

$$
\begin{gathered}
\int_{0}^{\infty} \int_{\mathbf{R}^{N}}|u-v| \psi(x)\left(\zeta_{\varepsilon}(t-\delta)-\zeta_{\varepsilon}(t-T)\right)\left(1-Y_{\theta}(|y|-R-\alpha(T-t))\right) d y d x d t \\
+\int_{0}^{\infty} \int_{\mathbf{R}^{N}}|u-\varphi| \Delta_{x} \psi(x)\left(Y_{\varepsilon}(t-\delta)-Y_{\varepsilon}(t-T)\right) \\
\cdot\left(1-Y_{\theta}(|y|-R-\alpha(T-t))\right) d y d x d y \\
+\int_{0}^{\infty} \int_{\mathbf{R}^{N}}[|f(u)-f(v)|-\alpha|u-v|] \psi(x)\left(Y_{\varepsilon}(t-\delta)-Y_{\varepsilon}(t-T)\right) \\
\cdot \zeta_{\theta}(|y|-R-\alpha(T-t))(\operatorname{sign} y) d x d y d t \geq 0
\end{gathered}
$$

Since $|f(u)-f(v)| \leq \alpha|u-v|$ and $Y_{\varepsilon}(t-\delta)-Y_{\varepsilon}(t-T) \geq 0$, we deduce that the last integral is $\leq 0$. Therefore, we get

$$
\begin{aligned}
& 0 \leq \int_{0}^{\infty} \int_{\mathbf{R}^{N}}|u-v| \psi(x)\left(\zeta_{\varepsilon}(t-\delta)-\zeta_{\varepsilon}(t-T)\right) \\
& \cdot\left(1-Y_{\theta}(|y|-R-\alpha(T-t))\right) d y d x d t \\
&+\int_{0}^{\infty} \int_{\mathbf{R}^{N}}|u-v| \Delta_{x} \psi(x)\left(Y_{\varepsilon}(t-\delta)-Y_{\varepsilon}(t-T)\right) \\
& \cdot\left(1-Y_{\theta}(|y|-R-\alpha(T-t))\right) d y d x d t
\end{aligned}
$$

Now we let $\theta$ tend to 0 . Applying Lebesgue's dominated convergence theorem we get

$$
\begin{aligned}
0 \leq & \int_{\mathbf{R}^{N-1}} \int_{K_{R, T}^{\varepsilon}}|u-v| \psi(x)\left(\zeta_{\varepsilon}(t-\delta)-\zeta_{\varepsilon}(t-T)\right) d x d y d t \\
& +\int_{\mathbf{R}^{N-1}} \int_{K_{R, T}^{\varepsilon}}|u-v|\left(\Delta_{x} \psi(x)\right)\left(Y_{\varepsilon}(t-\delta)-Y_{\varepsilon}(t-T)\right) d x d y d t
\end{aligned}
$$

with

$$
K_{R, T}^{\varepsilon}=\left\{(y, t) \in \mathbf{R} \times \mathbf{R}^{+}:|y| \leq R+\alpha(T-t), \delta-\varepsilon \leq t \leq T+\varepsilon\right\} .
$$

This can be equivalently rewritten as follows:

$$
\begin{aligned}
0 \leq & \int_{0}^{\infty}\left(\zeta_{\varepsilon}(t-\delta)-\zeta_{\varepsilon}(t-T)\right) \int_{\mathbf{R}^{N-1}} \int_{S_{t}} \psi(x)|u-v| d y d x d t \\
& +\int_{0}^{\infty}\left(Y_{\varepsilon}(t-\delta)-Y_{\varepsilon}(t-T)\right) \int_{\mathbf{R}^{N-1}} \int_{S_{t}} \Delta_{x} \psi(x)|u-v| d y d x d t
\end{aligned}
$$

where $S_{t}$ is as follows: $S_{t}=\{y \in \mathbf{R}:|y| \leq R+\alpha(T-t)\}$. Now,

$$
\begin{gathered}
\lim _{\varepsilon \rightarrow 0} \int_{0}^{\infty}\left(Y_{\varepsilon}(t-\delta)-Y_{\varepsilon}(t-T)\right) \int_{\mathbf{R}^{N-1}} \int_{S_{t}} \Delta_{x} \psi(x)|u-v| d y d x d t \\
\quad=\int_{\delta}^{T} \int_{\mathbf{R}^{N-1}} \int_{S_{t}} \Delta_{x} \psi(x)|u-v| d y d x d t
\end{gathered}
$$

and then clearly

$$
\begin{gathered}
\lim _{\delta \rightarrow 0} \lim _{\varepsilon \rightarrow 0} \int_{0}^{\infty}\left(Y_{\varepsilon}(t-\delta)-Y_{\varepsilon}(t-T)\right) \int_{\mathbf{R}^{N-1}} \int_{S_{t}} \Delta_{x} \psi|u-v| d y d x d t \\
=\int_{0}^{T} \int_{\mathbf{R}^{N-1}} \int_{S_{t}} \Delta_{x} \psi(x)|u-v| d y d x d t
\end{gathered}
$$


On the other hand, if we introduce the function $w: \mathbf{R}^{N-1} \times \mathbf{R}_{+} \mapsto \mathbf{R}$, defined by

$$
w(x, t)=\int_{S_{t}}|u(x, y, t)-v(x, y, t)| d y,
$$

and set $w_{\varepsilon}=w * \zeta_{\varepsilon}$ (convolution in the time variable), defined for $t \geq \varepsilon$, we have

$$
\begin{gathered}
\int_{0}^{\infty}\left(\zeta_{\varepsilon}(t-\delta)-\zeta_{e}(t-T)\right) \int_{\mathbf{R}^{N-1}} \psi(x) \int_{S_{t}}|u-v| d y d x d t \\
=\int_{\mathbf{R}^{N-1}}\left(w_{\varepsilon}(x, \delta)-w_{\varepsilon}(x, T)\right) \psi(x) d x
\end{gathered}
$$

Letting first, $\varepsilon \rightarrow 0$ and then $\delta \rightarrow 0$ we obtain that

$$
\begin{aligned}
\int_{\mathbf{R}^{n-1}} & \psi(x) \int_{|y| \leq R+\alpha T}\left|u_{0}(x, y)-\varphi_{0}(x, y)\right| d y d x \\
& -\int_{\mathbf{R}^{n-1}} \psi(x) \int_{|y| \leq R}|u(x, y, T)-v(x, y, T)| d y d x \\
= & \lim _{\delta \rightarrow 0} \lim _{\varepsilon \rightarrow 0} \int_{0}^{\infty}\left(\zeta_{\varepsilon}(t-\delta)-\zeta_{\varepsilon}(t-T)\right) \int_{\mathbf{R}^{N-1}} \int_{S_{t}} \psi(x)|u-v| d y d x d t
\end{aligned}
$$

From (3.10)-(3.12) we deduce that

$$
\begin{aligned}
\int_{\mathbf{R}^{N-1}} & \psi(x) \int_{|y| \leq R+\alpha T}\left|u_{0}(x, y)-v_{0}(x, y)\right| d y d x \\
& -\int_{\mathbf{R}^{n-1}} \psi(x) \int_{|y| \leq R}|u(x, y, T)-v(x, y, T)| d y d x \\
& +\int_{0}^{T} \int_{\mathbf{R}^{N-1}} \Delta_{x} \psi(x) \int_{S_{l}}|u-v| d y d x d t \geq 0
\end{aligned}
$$

Let us choose now $\varphi \in \mathscr{D}\left(\mathbf{R}^{N-1}\right)$ such that

$$
\varphi= \begin{cases}1 & \text { if }|x| \leq 1 \\ 0 & \text { if }|x| \geq 2\end{cases}
$$

and $0 \leq \varphi \leq 1$ when $1 \leq|x| \leq 2$. We take in (3.13), $\psi(x)=\varphi(x / \beta)$ and let $\beta \rightarrow \infty$. We obtain in this way

$$
\begin{aligned}
& \int_{\mathbf{R}^{N-1}} \int_{|y| \leq R}|u(x, y, T)-v(x, y, T)| d y d x \\
& \quad \leq \int_{\mathbf{R}^{N-1}} \int_{|y| \leq R+\alpha T}\left|u_{0}(x, y)-v_{0}(x, y)\right| d y d x,
\end{aligned}
$$

with completes the proof of Lemma 3.4.

Remark 3.5. Note that by taking limits in (3.10) as $R \rightarrow \infty$ we obtain the $L^{1}\left(\mathbf{R}^{N}\right)$-contraction property (1.3) for the entropy solutions of (1.1) with smooth initial data.

On the other hand, the comparison property can be easily obtained by the uniqueness property we have proved. Indeed, since every entropy solution of (1.1) with smooth initial data is the limit as $\varepsilon \rightarrow 0$ of the solutions of the 
regularized problem (2.2) and since by the Maximum Principle the comparison holds for (2.2), it also holds for (1.1).

\section{EXISTENCE AND UNIQUENESS IN $L^{1} \cap L^{\infty}$}

We complete in this section the proof of our main results by dealing with bounded and integrable initial data, possibly nonsmooth.

Existence. Given $u_{0} \in L^{1}\left(\mathbf{R}^{N}\right) \cap L^{\infty}\left(\mathbf{R}^{N}\right)$, we construct a sequence of smooth data $\left\{u_{0, n}\right\} \subset \mathscr{D}\left(\mathbf{R}^{N}\right)$, such that $\left\{u_{0, n}\right\}$ is uniformly bounded in $\mathbf{R}^{N}$ and

$$
u_{0, n} \rightarrow u_{0} \quad \text { in } L^{1}\left(\mathbf{R}^{N}\right) \text { as } n \rightarrow \infty .
$$

Let us denote by $u_{n}$ the entropy solution of (1.1) with data $u_{0, n}$. In view of (4.1) and the contraction (1.4) we conclude that the sequence $\left\{u_{n}\right\}$ converges in $C\left([0, \infty): L^{1}\left(\mathbf{R}^{N}\right)\right)$ to a function $u=u(x, y, t)$ as $n \rightarrow \infty$. In view of (1.6) the $u_{n}$ are also uniformly bounded in $L^{\infty}\left(\left(\mathbf{R}^{N}\right) \times(0, \infty)\right)$. By interpolation we deduce that

$$
u_{n} \rightarrow u \quad \text { in } C\left([0, \infty): L^{p}\left(\mathbf{R}^{N}\right)\right)
$$

for every $p \in[1, \infty)$. This allows us to pass to the limit in equation (1.1) and entropy condition (EC), which are of course satisfied by $u_{n}$. Thus, the limit is an entropy solution of (1.1). It also verifies the initial data $u_{0}$.

Uniqueness. Since the solutions are supposed to be bounded and belong to $C\left([0, \infty): L^{1}\left(\mathbf{R}^{N}\right)\right)$, proceeding as in $\S 3$ we may prove that for every pair of entropy functions $u$ and $v$ we have

$$
\int_{\mathbf{R}^{N}}|u(x, y, T)-v(x, y, T)| d x d y \leq \int_{\mathbf{R}^{N}}|u(x, y, t)-v(x, y, t)| d x d y
$$

whenever $0<t<T$. Taking limits as $t \rightarrow 0$ on the right-hand side we deduce the $L^{1}$-contraction property (1.4), hence uniqueness. The comparison principle is now immediate since it holds for the approximate problems.

\section{EXTENSIONS AND APPLICATIONS}

I. Since the main estimates are in fact $L^{1}$ bounds, the above method of approximation can be applied to initial data merely in $L^{1}\left(\mathbf{R}^{N}\right)$. There is however a difficulty, namely the fact that we have used the boundedness of the solutions for $t \geq \tau>0$. This property can be obtained as an a priori estimate for integrable data under some assumptions, and is known as the $L^{1}-L^{\infty}$ regularizing effect. In [EVZ, Lemma 2.2] we have the regularizing effect for solutions of $(0.2)$ if $f$ is of the form

$$
f(u)=|u|^{q-1} u, \quad \text { if } 1<q<2,
$$

and the proof stays unchanged for equation $(0.1)$ with the same convective nonlinearity.

II. There is even the possibility of taking measures as initial data. In fact, as the problem of a convective equation with partial diffusivity first arose in [EVZ], the question was to construct and characterize a fundamental solution of $(0.1)$, i.e. a solution having a Dirac mass as initial data. Such important development needs further work and is covered in [EVZ], where it is also shown that the solution has self-similar form. 
III. Clearly, there is a rather large class of equations of the form "conservation law plus viscosity" to which our approach can be extended, including cases like here where viscosity is not present in the convection directions, but also cases where they have common directions, as in

$$
u_{t}=\sum_{1}^{m} \frac{\partial^{2} u}{\partial x_{i}^{2}}+\sum_{1}^{m} \partial_{x_{i}}\left(g_{i}(u)\right)+\sum_{m+1}^{N} \partial_{x_{i}}\left(f_{i}(u)\right) .
$$

In this case the entropy condition we propose reads

$$
\begin{aligned}
\partial_{t} \mid u- & \psi\left|-\sum_{1}^{m} \frac{\partial^{2}}{\partial x_{i}^{2}}\right| u-\psi \mid \leq \sum_{1}^{m} \partial_{x_{i}}\left[\left(g_{i}(u)-\psi\right) \operatorname{sign}(u-\psi)\right] \\
+ & \sum_{m+1}^{N} \partial_{x_{i}}\left[\left(f_{i}(u)-\psi\right) \operatorname{sign}(u-\psi)\right]+\operatorname{sign}(u-\psi) \sum_{1}^{m} \frac{\partial^{2} \psi}{\partial x_{i}^{2}},
\end{aligned}
$$

with $\psi=\psi\left(x_{1}, \ldots, x_{m}\right)$.

Further investigation is needed to clarify the scope and usefulness of the concept of solution proposed. To our knowledge this is a novel treatment of Kruzhkov's entropy approach even in the standard case when viscosity applies isotropically in all directions.

\section{Comparative Discussion}

Our results can be viewed as providing a unified method of treating the question of unique characterization of solutions of scalar conservation laws involving viscosity in different forms or no viscosity at all. Up to this moment, and to our knowledge, the question has been treated in detail in the extreme cases of (i) equations having complete viscosity, and (ii) those having no viscosity at all.

In the case of complete viscosity a typical uniqueness is based on Kato's inequality $[\mathrm{Ka}]$ in the form

$$
\Delta|u-v| \leq \operatorname{sign}(u-v) \Delta(u-v) .
$$

The regularity implied by the diffusion term allows to consider time derivatives as functions so that $\partial_{t}|u-v|=\operatorname{sign}(u-v) \cdot(u-v)_{t}$, and the uniqueness proof consists in integrating $\partial|u-v|_{t}$ in $Q$, use the equation and Kato's inequality to conclude that $|u-v|=0$ a.e.

This is no more possible for the nonviscous case, hence the need for the extra entropy condition, like Kruzhkov's, which is by the way automatically fulfilled for a completely viscous law. Thus, no extra conditions need to be imposed on the solutions in this case.

\section{ACKNOWLEDGMENTS}

This work was completed while the third author visited the Institute of Mathematics and its Applications, Minnesota. The first author was partially supported by DGICYT Project PB90-0245, the second by EEC Grant SC1-0019C(TT) and DGICYT Project PB90-0218 and the third by DGICYT Project PB90-0245 and by EEC Grant SCI-CT91-0732. 


\section{REFERENCES}

[EVZ] M. Escobedo, J. L. Vazquez, and E. Zuazua, A diffusion-convection equation in several space dimensions, preprint.

[EZ] M. Escobedo and E. Zuazua, Large-time behaviour of convection-diffusion equations in $\mathbf{R}^{N}$, J. Funct. Anal. 100 (1991), 119-161.

[GR] E. Godlewski and P. A. Raviart, Hyperbolic systems of conservation laws, SMAI 3/4, Ellipses-Edition Marketing, Paris, 1991.

[Ka] T. Kato, Schrödinger operators with singular potentials, Israel J. Math. 13 (1972), 135-148.

[Kr] S. Kruzhkov, First-order quasilinear equations in several independent variables, Mat. USSRSb. 10 (1970), 217-243.

Departamento de Matemáticas, Universidad del Pais Vasco, Apartado 644, 48080 BilBAO, SPAIN

E-mail address: mtpesmam@lg.ehu.es

Departamento de Matemáticas, Universidad Autónoma de Madrid, 28049 Madrid, SPAIN

E-mail address: vazquez@rm1.sdi.uam.es

Departamento de Matemática Aplicada, Universidad Complutense, 28040 Madrid, SPAIN

E-mail address: zuazua@mat.ucm.es 